\title{
The use of electron scattering for studying atomic momentum distributions: The case of graphite and diamond
}

\author{
M. Vos, ${ }^{1, a)}$ R. Moreh, ${ }^{2}$ and K. Tőkési ${ }^{3}$ \\ ${ }^{1}$ Atomic and Molecular Physics Laboratories, Research School of Physics and Engineering, Australian \\ National University, Canberra, Australian Capital Territory 0200, Australia \\ ${ }^{2}$ Physics Department, Ben-Gurion University of the Negev, Beer-Sheva 84105, Israel \\ ${ }^{3}$ Institute of Nuclear Research of the Hungarian Academy of Sciences (ATOMKI), H-4001 Debrecen, P.O. Box \\ 51, Hungary, EU
}

(Received 24 March 2011; accepted 13 June 2011; published online 12 July 2011)

\begin{abstract}
The momentum distributions of $\mathrm{C}$ atoms in polycrystalline diamond (produced by chemical vapor deposition) and in highly oriented pyrolitic graphite (HOPG) are studied by scattering of $40 \mathrm{keV}$ electrons at $135^{\circ}$. By measuring the Doppler broadening of the energy of the elastically scattered electrons, we resolve a Compton profile of the motion of the $\mathrm{C}$ atoms. The aim of the present work is to resolve long-standing disagreements between the calculated kinetic energies of carbon atoms in HOPG and in diamond films and the measured ones, obtained both by neutron Compton scattering (NCS) and by nuclear resonance photon scattering (NRPS). The anisotropy of the momentum distribution in HOPG was measured by rotating the HOPG sample relative to the electron beam. The obtained kinetic energies for the motion component along, and perpendicular to, the graphite planes were somewhat higher than those obtained from the most recent NCS data of HOPG. Monte Carlo simulations indicate that multiple scattering adds about $2 \%$ to the obtained kinetic energies. The presence of different isotopes in carbon affects the measurement at a $1 \%$ level. After correcting for these contributions, the kinetic energies are 3\%-6\% larger than the most recent NCS results for HOPG, but $15 \%-25 \%$ smaller than the NRPS results. For diamond, the corrected direction-averaged kinetic energy is $\approx 6 \%$ larger than the calculated value. This compares favorably to the $\approx 25 \%$ discrepancy between theory and both the NCS and NRPS results for diamond. (C) 2011 American Institute of Physics. [doi:10.1063/1.3607993]
\end{abstract}

\section{INTRODUCTION}

There are very few techniques that can determine directly the kinetic energy of atoms bound to solids or molecules. Two such techniques are neutron Compton scattering (NCS) (Refs. 1-5) and nuclear resonance photon scattering (NRPS). ${ }^{6,7}$ Both techniques rely on Doppler broadening caused by the momentum of the scattering atom.

In NCS the energy transferred by the neutron to the target atom depends on its atomic momentum. For large-angle deflection of high-energy neutrons $(\approx 10 \mathrm{eV})$ from a nucleus, it appears as if the collision is between free particles (impulse approximation applies). The observed neutron energy distribution contains the Compton profile of the momentum distribution of the scatterer before the collision. ${ }^{1}$

In the NRPS method, described in detail elsewhere, 6,7 one creates a situation in which the scattering photon cross section (for $\mathrm{MeV}$ photons) is related to the Doppler broadening of the nuclear level. The photon energies in the NRPS method must be high enough to excite nuclear levels of the target atoms.

Two carbon-based materials, chemical vapor deposition (CVD) diamond and highly oriented pyrolitic graphite (HOPG), have been studied by NCS as well as NRPS. The

\footnotetext{
a) Author to whom correspondence should be addressed. Electronic mail: maarten.vos@anu.edu.au.
}

C-atoms in HOPG are characterized by two different meansquare momenta (or directional kinetic energies) corresponding to the anisotropic directions: parallel and perpendicular to the planes of HOPG. Hence, in NRPS two different cross sections are obtained for alignment of the parallel and perpendicular direction with respect to the photon beam. In NCS different spectra are obtained, depending on the alignment of the momentum transfer with these crystallographic directions. Diamond is a much more isotropic system, and anisotropies would be hard to measure. Moreover, both NRPS and NCS studies used polycrystalline CVD diamond and one obtains the direction-averaged kinetic energy. Surprisingly, clear deviations between both experimental results were found for both CVD diamond and HOPG. Moreover, neither of the results was in good agreement with theory. This is not understood because the methods are rather direct, and the kinetic energy calculations are straightforward and based on wellestablished phonon dispersion relations.

The situation is completely different for hexagonal $\mathrm{Zn}$ metal. Here, a different version of the NRPS method was used $^{8}$ compared to the one for carbon. ${ }^{6,7}$ A comparison of this NRPS method ${ }^{8}$ and the NCS method ${ }^{9}$ was made for this highly anisotropic system, studied at low temperatures $(\approx 10$ $\mathrm{K})$. The corresponding zero-point kinetic energies of the $\mathrm{Zn}$ atoms along and perpendicular to the hexagonal planes were measured, and the magnitude of the anisotropy obtained by both techniques was in excellent agreement. 
Recently, it has become clear that keV electron scattering at high momentum transfer is another technique which depends on Doppler broadening ${ }^{10-13}$ and could be used as a powerful tool for studying binding properties of low $Z$ elements, such as $\mathrm{H}, \mathrm{D}$, and $\mathrm{C}$. The instantaneous motion of the scattering atom causes a Doppler broadening of the energy of elastically scattered electrons. By using an electron spectrometer of very high resolution, it was possible to measure this broadening and to deduce the instantaneous kinetic energies of the scattering atoms. The Doppler broadening needs to be at least of nearly the same magnitude as the instrumental resolution of the spectrometer in order to be able to determine the kinetic energy of the scattering atoms. For strongly bound, light atoms the major part of their kinetic energy, even at room temperature, is contributed by their zero-point motion and hence the e-scattering technique may be used for measuring the zero-point kinetic energies of $\mathrm{C}$ atoms in diamond, graphite, and $\mathrm{C}$-containing molecules. In fact, a recent e-scattering study from $\mathrm{H}$-containing gases, such as $\mathrm{CH}_{4}$, has shown that this technique yields the Doppler broadenings of the elastically scattered lines separately from both the $\mathrm{H}$ and C-atoms. ${ }^{14}$ The separation energy between the $\mathrm{H}$ - and $\mathrm{C}$ peaks is reasonably well described using the impulse approximation in which all the particles involved in the scattering process are assumed to be free. However, the shape of the corresponding peaks could only be explained by modifying the impulse approximation to include final state effects. These deviations of the impulse approximation are expected to decrease with increasing momentum transfer.

Can this technique be used to shed light on the disagreement between calculated and measured kinetic energies? This is the focus of this paper. We employ for this purpose the same electron energy of $40 \mathrm{keV}$ but use a larger scattering angle, $135^{\circ}$, compared to $45^{\circ}$ used in our previous study of graphite. ${ }^{11}$ As a consequence, the momentum transfer is 2.4 times larger, and the Doppler broadening is increased proportionally. Hence, the intrinsic width of the carbon peaks can be determined much more accurately, and our results are of similar quality as those obtained by neutron Compton scattering. The larger momentum transfer also means that deviations of the impulse approximation, already a small effect in Ref. 11, are further reduced and can be neglected for the purpose of this paper.

\section{BACKGROUND}

An electron with energy $E_{0}$ and momentum $\mathbf{k}_{0}$ scatters from a nucleus and is detected after scattering over an angle $\theta$ with momentum $\mathbf{k}_{1}$. We assume for now that the deflection of the incoming electron is due to a collision with a single target atom. The momentum transfer $\mathbf{q}$ in the collision is thus given by $\mathbf{q}=\mathbf{k}_{1}-\mathbf{k}_{0}$. At high momentum transfer, the impulse approximation is valid: it appears that the electron scatters from a free atom with momentum $\mathbf{p}$. The energy loss of an electron after quasi-elastic scattering from a nucleus with mass $M$ is then given by

$$
E_{r}=\frac{q^{2}}{2 M}+\frac{\mathbf{q} \cdot \mathbf{p}}{M} .
$$

The first term is the recoil for scattering from a stationary particle. The second term is the Doppler broadening due to the motion of the target atom. As a value of $\mathbf{p}$ is as likely as a value of $-\mathbf{p}$, the average recoil energy $\overline{E_{r}}$ is given by $q^{2} / 2 M$. The second moment $\mu_{2}$ of the recoil energy distribution for scattering from a target with momentum distribution $f(\mathbf{p})$ is thus given by

$$
\begin{aligned}
\mu_{2} & =\int_{p}\left(E_{r}-\overline{E_{r}}\right)^{2} f(\boldsymbol{p}) d \boldsymbol{p}=\int_{p}\left(\frac{\mathbf{q} \cdot \mathbf{p}}{M}\right)^{2} f(\boldsymbol{p}) d \boldsymbol{p} \\
& =4 \overline{E_{r}} \int_{p} \frac{p_{q}^{2}}{2 M} f(\boldsymbol{p}) d \boldsymbol{p},
\end{aligned}
$$

with $p_{q}$ the component of $\mathbf{p}$ along $\mathbf{q}$. For an isotropic solid $\int_{p}\left(p_{q}^{2} / 2 M\right), f(\boldsymbol{p}) d \boldsymbol{p}$ is just one-third of the total average kinetic energy of an atom $\left(\overline{E_{k i n}}\right)$ and the standard deviation $\sigma=\sqrt{\mu_{2}}$ may be written as

$$
\sigma=\sqrt{\frac{4}{3} \overline{E_{r}} \overline{E_{k i n}}} .
$$

For an anisotropic solid, such as graphite, the observed width is directly related to the mean kinetic energy associated with the motion component along $\boldsymbol{q}$. Thus, one can determine both $\overline{E_{k i n}^{p}}$ and $\overline{E_{k i n}^{c}}$ (for $\boldsymbol{q}$ along and normal to the graphite planes, respectively) from

$$
\overline{E_{k i n}^{c, p}}=\frac{\sigma_{c, p}^{2}}{4 \overline{E_{r}}} .
$$

It is often the custom to refer to the energy width of a feature in terms of effective temperature $T_{e}$, which is equal to the temperature of a classical gas that would have the same Doppler broadening as the measured line. This is done, e.g., in Ref. 7, and we use this convention. Other authors (e.g., Ref. 2) express energy in terms of temperature $(E=k T)$. In this language, the kinetic energy of a classical gas at a temperature $T$ is $(3 / 2) T_{e}$.

In these measurements, the width is determined by the distribution of the component of the atoms momentum along $q$. It can be derived from the phonon spectrum along this direction, $g(v)_{q}$,

$$
\int_{p} \frac{\left(p_{q}\right)^{2}}{2 M} f(\boldsymbol{p}) d \boldsymbol{p}=0.5 \times \frac{\int_{0}^{v_{\max }} g(v)_{q} h v \alpha d v}{\int_{0}^{v_{\max }} g(v)_{q} d v},
$$

with $\alpha=\left[\left(e^{h v / k T}-1\right)^{-1}+(1 / 2)\right]$. The factor 0.5 in Eq. (5) reflects the fact that only half the harmonic oscillator energy is kinetic energy, the remaining half is potential energy.

For graphite, $g(v)_{q}$ has been calculated for q along and perpendicular to the planes. ${ }^{15}$ For isotropic materials such as diamond, the outcome of Eq. (5) does not depend strongly on the direction of $\boldsymbol{q}$.

\section{EXPERIMENTAL DETAILS}

The experiment was described in detail in Refs. 16 and 17 . The only significant change in the current setup is the employment of a larger scattering angle of $135^{\circ}$. HOPG samples were cleaved just before inserting them into the vacuum chamber, and a small amount of Au was deposited on 
the HOPG film. Due to the different masses of $\mathrm{Au}$ and $\mathrm{C}$, electrons scattered from either element appear at different energy losses (see Eq. (1)). This is the electron analog of (ion) Rutherford backscattering (RBS). For this reason, we refer to this technique often as electron Rutherford backscattering (ERBS). ${ }^{18}$ These samples were clean, and no impurities other than Au were observed in the ERBS spectra.

CVD diamond is the term applied to $s p^{3}$-bonded carbon grown by chemical vapor deposition. It is usually polycrystalline. In our study we used a CVD-diamond sample of dimensions of $5.0 \mathrm{~mm} \times 5.0 \mathrm{~mm} \times 0.3 \mathrm{~mm}$ which was laser cut from a $25 \mathrm{~mm}$ diameter self-supporting plate produced by CVD, as described in Ref. 19. It was measured without any surface modification but showed some heavy impurities. It was not attempted to clean this sample, as sputtering and/or annealing has a tendency to change the nature of the chemical bonding of the atoms in the surface layer. It should be stressed that these electron scattering techniques are somewhat surface sensitive (e.g., the inelastic mean free path for diamond is estimated to be $64 \mathrm{~nm},{ }^{20}$ which means that the majority of the electrons contributing to the elastic peak scatter at a depth of $30 \mathrm{~nm}$ or less.)

\section{RESULTS}

Examples of spectra obtained from electrons scattering from $\mathrm{HOPG}$ with an $\approx 0.3 \AA$ thick Au layer are shown in Fig. 1. The spectra shown here were obtained in two different geometries. In one geometry, the momentum transfer was along the surface normal ( $c$-axis), in the second geometry, the momentum transfer is at $45^{\circ}$ angle with the surface normal. In both cases we see two completely resolved peaks. The narrow one, at low energy loss, is associated with electrons scattering from Au. The second broader peak, at larger energy loss, is due to electrons scattered from carbon. The positions of these two peaks, based on Eq. (1) and at $40 \mathrm{keV}$ and $135^{\circ}$ scattering angle, should be at $0.40 \mathrm{eV}(\mathrm{Au})$ and $6.48 \mathrm{eV}(\mathrm{C})$ energy loss. As the zero of our energy scale is not known with sub-eV resolution, we align the Au peak with $0.4 \mathrm{eV}$ energy loss. The

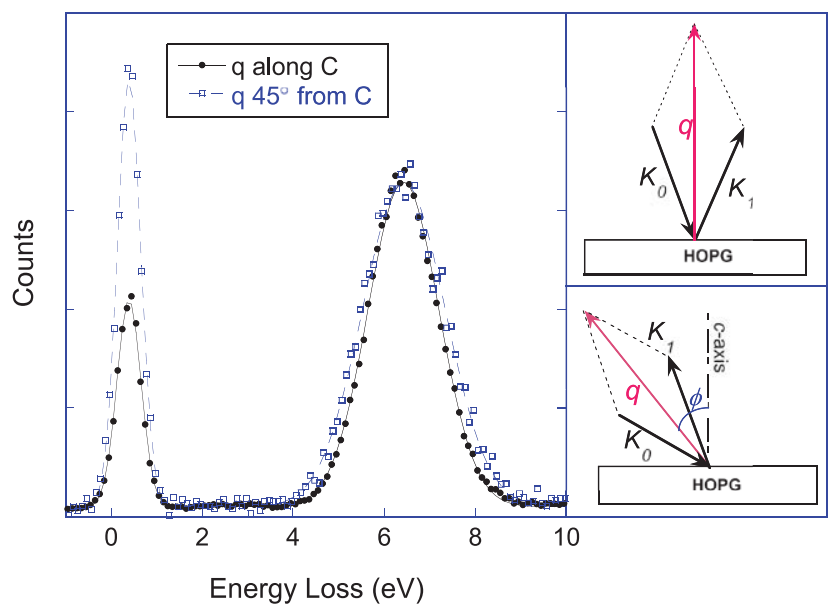

FIG. 1. The left panel shows spectra of a HOPG sample with some Au deposited on the surface for $\boldsymbol{q}$ along the $c$-axis $(\phi=0)$, for $\phi=45^{\circ}$. The right panel illustrates the measurement geometries used. second peak appears then indeed close to $6.5 \mathrm{eV}$, as expected for $\mathrm{C}$. The Au peak width $(\sigma)$ is $0.25 \mathrm{eV}$, much less than the $\mathrm{C}$ peak width. For $\mathrm{Au}$, the Doppler broadening is not expected to be resolved due to its large atomic mass (see Eq. (1)). Thus, the Au width is taken to be the experimental resolution. The carbon peak width is larger, and depends on the measurement geometry. The configuration with $\boldsymbol{q}$ along the $c$-axis has the smallest width. Thus, the anisotropy of the atomic motion in HOPG is resolved.

In the measurement with $\boldsymbol{q}$ directed $45^{\circ}$ away from the $c$-axis, the incoming beam is quite glancing with the surface. This makes the measurement more surface sensitive, as is evident from the larger size of the Au peak (at the surface) under these conditions. Measurements under even more glancing conditions (and hence with $\boldsymbol{q}$ at an even larger angle with the $c$-axis) were not done as the metal clip retaining the graphite sample started to interfere with the measurement.

The largest width is expected for $\boldsymbol{q}$ perpendicular to the $c$-axis. This geometry could, in principle, be realized using a thin HOPG sample in a transmission geometry. However, both incoming and outgoing beams would be rather glancing (22.5 $5^{\circ}$ away from the surfaces), and even for very thin HOPG samples (e.g., $150 \AA$ ), the length of the trajectories inside the sample would still be rather long $\left(t / \sin \left(22.5^{\circ}\right)\right.$ with $t$ the sample thickness ) making such an experiment extremely challenging, and it was not attempted.

As the two peaks are well separated, and there is no significant background, it is very straightforward to fit the spectra with two Gaussians, one for $\mathrm{Au}$ and the other for $\mathrm{C}$. The statistical uncertainty in the width extracted from these fits is very small, and by measuring a series of angles, we obtain the variation in $\mathrm{C}$ width with angle. This width was corrected for experimental resolution by subtracting the observed $\mathrm{Au}$ width in quadrature. The resulting widths are shown in Fig. 2. We can fit the experimental data with the expected angular dependence of the width:

$$
\sigma(\phi)=\sigma_{p} \sin ^{2}(\phi)+\sigma_{c} \cos ^{2}(\phi),
$$

with $\phi$ as the angle between $\boldsymbol{q}$ and the $c$-axis, and $\sigma_{p}$ and $\sigma_{c}$ as the $\mathrm{C}$ peak width for $\boldsymbol{q}$ along the planes and along the

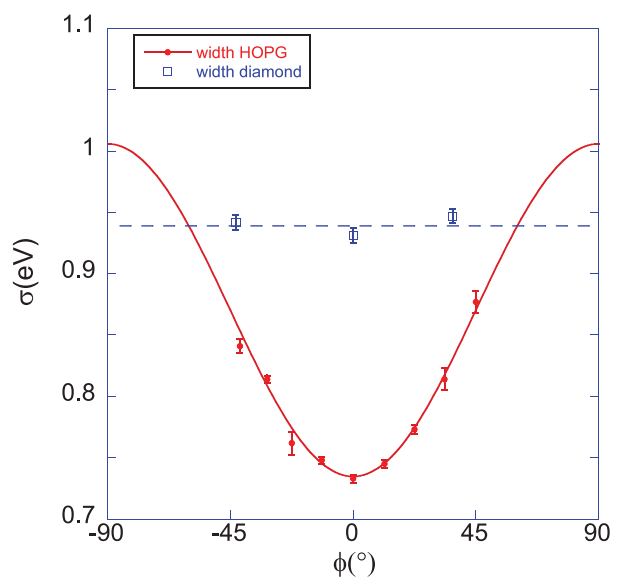

FIG. 2. The dependence of the elastic peak width on $\phi$, the angle between the momentum transfer direction and the surface normal, for both HOPG and amorphous diamond. The full line is a fit, based on Eq. (6). 
TABLE I. Effective temperature (K) as calculated and measured for graphite. $T_{p}$ and $T_{c}$ refer to the effective temperatures along and normal to the graphite planes, respectively. $T_{e}$ is the average temperature, obtained by $T_{e}=\left(2 T_{p}+T_{c}\right) / 3$. The values in column marked "ECS, as measured" refer to the present measurements, as measured, and the values in column "ECS corrected" are the results of the same measurement after correction for multiple scattering and isotope effects.

\begin{tabular}{|c|c|c|c|c|c|c|c|c|c|}
\hline & \multicolumn{3}{|c|}{ Theory } & \multirow{2}{*}{$\begin{array}{c}\text { NRPS } \\
7 \\
\end{array}$} & \multicolumn{3}{|c|}{ NCS } & \multirow{2}{*}{$\frac{\text { ECS }}{\text { As measured }}$} & \multirow{2}{*}{$\begin{array}{c}\text { ECS } \\
\text { corrected }\end{array}$} \\
\hline & 21 & 22 & 15 & & 3 & 4 & 2 & & \\
\hline$\overline{T_{p}}$ & & & 798 & $975 \pm 80$ & $1050 \pm 80$ & $990 \pm 55$ & $855 \pm 3$ & $906 \pm 18$ & $880 \pm 18$ \\
\hline$T_{c}$ & & & 548 & $607 \pm 37$ & $550 \pm 30$ & $552 \pm 30$ & $441 \pm 2$ & $483 \pm 10$ & $468 \pm 10$ \\
\hline$T_{e}$ & 711 & 759 & 715 & $863 \pm 68$ & $883 \pm 63$ & $834 \pm 63$ & $717 \pm 2$ & $765 \pm 15$ & $743 \pm 15$ \\
\hline
\end{tabular}

$c$-axis, respectively. In this way, we obtain an estimate of $\sigma_{p}$. Using Eq. (2), we can now obtain an estimate of the kinetic energy along and perpendicular to the $c$-axis. The obtained values (referred to as $E_{k i n}^{p}$ and $E_{k i n}^{c}$ ) are shown in Table I and are compared with the previously obtained values for these quantities.

The quality of the fit of the angular dependence of the width is quite good, and deviations between the measured values and the fit are of the order of the statistical uncertainty of the width of the C peak. A similar plot, using the NCS technique, was produced by Fielding et $a l^{2}$ Here, there are significantly more data points, as many detectors and several sample orientations were used. However, the deviation between the observed width and the fit is, for the neutron experiment in many cases, much larger than the purely statistical uncertainty of each measurement point.

In Fig. 3, we show a spectrum obtained from the CVDdiamond sample after deposition of $\approx 0.3 \AA$ of Au. Besides the strong, sharp peak due to $\mathrm{Au}$, we also observe a smaller, but broader peak due to "medium $Z$ " impurities. The spectrum was fitted with three Gaussians: one for $\mathrm{Au}$, one for $\mathrm{C}$, and one for the "impurity." An Au-C separation of $6.03 \mathrm{eV}$ was obtained, very close to the expected value of $6.09 \mathrm{eV}$ expected for $40 \mathrm{keV}$ electrons. This means that there is no significant charging of the diamond film, and also that possible oxygen contamination of the sample does not contribute significantly to the peak attributed to $\mathrm{C}$, as the energy loss for scattering of $\mathrm{O}$ is $\approx 1.5 \mathrm{eV}$ less than for scattering from $\mathrm{C}$.

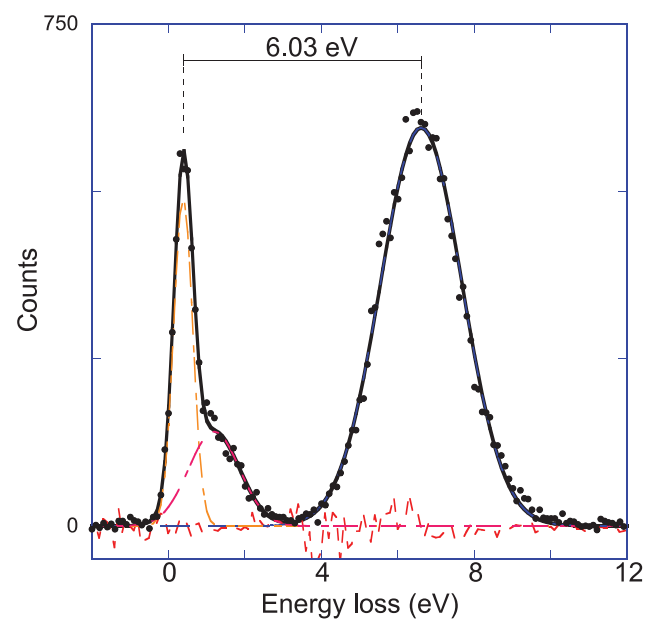

FIG. 3. Spectrum of $40 \mathrm{keV} \mathrm{e^{- }}$ scattered over $135^{\circ}$ from a CVD-diamond sample with $\approx 0.3 \AA$ Au deposited on the surface.
The obtained width of this sample is plotted in Fig. 2 as well. The measurement was repeated for three different orientations of the momentum transfer relative to the surface normal. Considering the error bar, the outcome of these measurements are consistent with no dependence of the width on the sample orientation, but, based on these experimental data, a very small dependency cannot be excluded.

\section{DISCUSSION}

\section{A. Graphite}

It is of interest to note that the results for HOPG (Table I) reveal huge deviations between the measured and calculated kinetic energies in both the parallel and the direction normal to the graphene planes. There are also large deviations between the results of the three NCS measurements. In fact the results of Ref. 2 indicate that in the normal direction, the predicted $E_{k i n}^{c}$ is $24 \%$ higher than the measured one, while $E_{k i n}^{p}$ is lower than the measured one by $7 \%$. Moreover, Table I shows that the deviations between the various measurements are also very large: In the measurement of Ref. 3, the measured $E_{k i n}^{p}$ was higher than the calculated value by $32 \%$. The present ECS results for HOPG show that the predicted $E_{k i n}^{c}$ is $13 \%$ higher than the measured one, while $E_{k i n}^{p}$ is lower than the measured one by $13 \%$; thus, the deviations in this case are more moderate.

It appears that the kinetic energy obtained from the calculated phonon spectrum along the $c$-axis in HOPG was reproduced experimentally by the older NCS results, ${ }^{3,4}$ but neither by the present study nor by that of the more recent NCS work. ${ }^{2}$ The situation along the direction parallel to the graphene planes is not any better as all measured values depart substantially from the calculated one. ${ }^{15}$

The direction-averaged kinetic energy of Young and Koppel ${ }^{15}$ of $715 \mathrm{~K}$ can be compared with other calculated values of $759 \mathrm{~K}$ (Ref. 22) and $711 \mathrm{~K} .{ }^{21}$ Thus it must be borne in mind that the three calculations based on the graphite phonon spectra (Refs. 15, 21, and 22) produce $C$ total kinetic energy values differing from each other by up to $7 \%$. The spread in experimentally determined values is several times larger.

In addition, the results for non-oriented graphite seem also to depart from each other by $30 \%$. This difference is more pronounced when one compares the more recent results of Ref. 2 with the older ones.

Deviations between the various experimental results are considerably larger than expected based on the error bars quoted by the authors. The theoretically obtained value of 
Ref. 22 seem to favor the present result, while that of Refs. 15 and 21 favors the latest NCS data. Thus, it is hard to decide which method is the most accurate in determining the mean kinetic energy for the different directions in HOPG.

\section{B. CVD diamond}

The value for the kinetic energies of the C-atoms in CVD diamond, obtained directly from the observed peak width (only corrected for energy resolution), using the e-scattering technique (see Table II), is close to within $9 \%$ of the value calculated from the phonon spectrum of diamond, ${ }^{23-25}$ and is the first technique which produced such a relatively close result to the predicted one. This is very significant in view of the fact that the present result is $20 \%$ lower than that measured by the other two methods: the NCS (Ref. 5) and the NRPS. ${ }^{6,7}$

\section{CORRECTIONS OF RAW ECS RESULTS}

The fact that the present uncorrected ECS result is $\approx 9 \%$ higher than the predicted value for diamond and $\approx 6 \%$ higher than the most recently measured NCS values for HOPG (Ref. 2) could be taken as an indication that there are additional broadening mechanisms at work in the ECS case. Due to the quadratic nature of Eq. (4), a 6\%-9\% discrepancy could be explained, if other mechanisms add only 3\%-4.5\% to the observed $\mathrm{C}$ width. Two such mechanisms, multiple scattering, and the presence of different isotopes will be discussed in Secs. VI A and VI B.

\section{A. Corrections due to multiple scattering}

A candidate for such a broadening mechanism is multiple scattering. Multiple scattering in ECS has been studied both theoretically ${ }^{26}$ and by Monte Carlo simulations, ${ }^{27}$ and the conclusion of both approaches was that multiple scattering is present but does not affect the outcome much. As only small corrections are required, we decided to do Monte Carlo simulations, employing the actual geometry of the experiment, to see if multiple scattering could cause a small increase in peak width.

Monte Carlo simulations of electron transport in solids are, in general, based on the stochastic description of the scattering processes. The electrons are assumed to follow a classical "zigzag" trajectory. At scattering points, the electron changes its direction (elastic collision) or its energy and direction (inelastic collision). In our simulations (for details see Ref. 27), both elastic and inelastic scattering events were taken into account. However, after the first inelastic collision

TABLE II. Effective temperature $(\mathrm{K})$ as calculated and measured for diamond, further similar to Table I.

\begin{tabular}{cccccccc}
\hline \hline \multicolumn{3}{c}{ Theory } & & \multicolumn{5}{c}{ Experiment } \\
\cline { 1 - 4 } \cline { 5 - 7 } 23 & 24 & 25 & & NRPS (Ref. 7) & NCS (Ref. 5) & ECS raw & ECS corrected \\
\hline 725 & 718 & 721 & & $967 \pm 30$ & $934 \pm 25$ & $789 \pm 25$ & $767 \pm 25$ \\
\hline \hline
\end{tabular}

occurred, the simulation of that trajectory was abandoned, as it could not contribute to the elastic peak anymore.

The path length between two scattering events $s$ is calculated using a random number $R$, as $s=-\lambda \ln (R)$. Here, $\lambda$ is the total mean free path obtained from the elastic $\left(\lambda_{e}\right)$ and inelastic $\left(\lambda_{i}\right)$ mean free path using

$$
\frac{1}{\lambda}=\frac{1}{\lambda_{e}}+\frac{1}{\lambda_{i}} .
$$

We assume that the velocity distribution of the target atoms in the sample is isotropic, and that the kinetic energy is described by the Maxwell-Boltzmann distribution:

$$
P(\varepsilon) d \varepsilon=\frac{3 \sqrt{3}}{\sqrt{2 \pi}} \frac{\sqrt{\varepsilon}}{\bar{\varepsilon}}^{3 / 2} \exp \left(-\frac{3 \varepsilon}{2 \bar{\varepsilon}}\right) d \varepsilon,
$$

where $\bar{\varepsilon}$ is the average kinetic energy of the atoms in the given layer. We used $\bar{\varepsilon}=108 \mathrm{meV}$ for the average kinetic energy of the $\mathrm{C}$ atoms. This value corresponds to the value obtained from the neutron scattering experiments, ${ }^{5}$ and appears to describe electron spectroscopy measurements at smaller momentum transfer quite well. . $^{10,28}$

In the simulation, a scattering angle was obtained based on the differential elastic cross section and a random number; the corresponding recoil energy was calculated according to Eq. (1). The momentum of the scattering atom, which appears in Eq. (1), was chosen by Monte Carlo techniques using the appropriate Maxwell-Boltzmann distribution and another random number. In the simulation, a small fraction of the impinging electrons leaves the crystal with a momentum directed towards the analyzer. For these trajectories, we sum all the recoil energies and in this way we obtain the simulated ERBS spectrum. Moreover, we keep track of the fact if the electron scattered from a surface $\mathrm{Au}$ atom, $\mathrm{C}$ atoms, or both $\mathrm{Au}$ and $\mathrm{C}$ atoms. In this way, three energy distributions of elastically backscattered electrons were obtained corresponding to scattering only of carbon, only of gold, and mixed scattering (deflected both from carbon and gold).

The cross sections for backscattering at $135^{\circ}$ and $40 \mathrm{keV}$ are very small. In order to acquire sufficient statistics in a finite time, we studied the configuration with the highest symmetry. We choose the incoming beam along the surface normal. All directions at a $45^{\circ}$ cone with the surface normal are equivalent (and correspond to scattering over $135^{\circ}$ ), and we collected the energy of all electrons that appear within $1.0^{\circ}$ of this direction (in the experiment, the angular resolution is several times better). This means that the mean recoil energy varied from $6.438 \mathrm{eV}$ (scattering over $134^{\circ}$ ) to $6.532 \mathrm{eV}$ (scattering over $135^{\circ}$ ). Note that in the experiment only a small fraction of this cone is covered by the analyzer. To further simplify the simulation, we studied the case of amorphous carbon. We expect that broadening due to multiple scattering for amorphous carbon and HOPG, or polycrystalline diamond films to be very similar. More details of the simulations are given in Ref. 27.

A total of $10^{11}$ trajectories were simulated, for a sample with two layers ( $0.3 \AA \mathrm{Au}$ on an infinitely thick $\mathrm{C}$ target). The simulated spectra show two peaks, just as the experiment, due to scattering from $\mathrm{Au}$ and $\mathrm{C}$. However, in the simulations, it is clear that a small fraction of the electrons scattered from $\mathrm{C}$ 

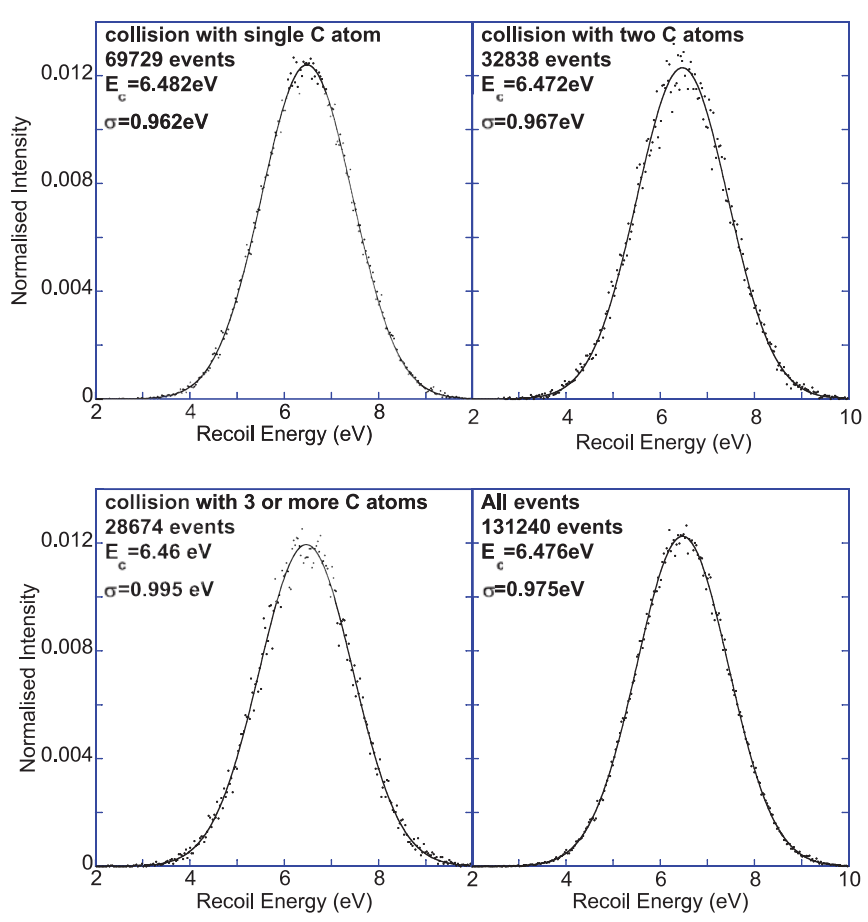

FIG. 4. Simulated spectra of electrons scattered from amorphous carbon. The results are plotted for the case of interaction with a single $\mathrm{C}$ atom only (top left), interaction with two $\mathrm{C}$ atoms (top right), and three or more $\mathrm{C}$ atoms (bottom left). The sum of all events is plotted in the bottom right.

atoms was deflected slightly by a Au atom. The separation of the simulated $\mathrm{Au}$ and $\mathrm{C}$ peaks was $6.08 \mathrm{eV}$, very close to the calculated single-scattering value of $6.09 \mathrm{eV}$.

Of the simulated $10^{11}$ trajectories, only 179064 trajectories (i.e., one for every $\approx 56000$ incoming electrons) left the sample in a $45^{\circ} \pm 1$ cone around the surface normal, taken to be the detector without any inelastic excitations (i.e., contribute to the elastic peak). Of these electrons, 131240 electrons scattered from $\mathrm{C}$ atoms only, 45806 electrons scattered from Au atoms only, and 2014 electrons scattered from both $\mathrm{Au}$ and $\mathrm{C}$ atoms.

We now focus on the 131240 cases where the electrons were scattered into the analyzer from carbon atoms only. The corresponding Doppler broadening $\sigma$ was $0.966 \mathrm{eV}$. These events were further sorted in different groups: electrons that had scattered from a single $\mathrm{C}$ atom, scattered from two $\mathrm{C}$ atoms, or scattered from three or more $\mathrm{C}$ atoms. The results are plotted in Fig. 4. For single scattering trajectories, the results should be very close to that expected based on the input data. The mean energy loss of 6.482(3) eV was obtained, very close to the mean value expected for this angular range, and the obtained Doppler broadening $(\sigma)$ of 0.962(2) $\mathrm{eV}$ was slightly smaller than the nominal value of $0.966 \mathrm{eV}$ for our assumed energy distribution of the $\mathrm{C}$ atoms. Thus, the additional broadening due to the finite opening angle assumed is (as expected) too small to influence the observed width.

The subset formed by particles that scattered from two carbon atoms consisted of $N=32838$ cases, considerably smaller than the subset of single scattered trajectories $(N=69729)$. The mean recoil energy of this subset was slightly reduced $(6.472(5) \mathrm{eV})$, and the width $0.967(3) \mathrm{eV}$ slightly increased.

Finally, the subset of particles with three or more interactions with $\mathrm{C}$ atoms $(N=28674)$ showed a further reduction in mean recoil energy $(6.463(5) \mathrm{eV})$, and again in a small further increase in width $(0.995(4) \mathrm{eV})$.

The combined result has a mean recoil energy of $6.476(0.003) \mathrm{eV}$ and a width of $0.975(0.002) \mathrm{eV}$. Thus, from these Monte Carlo simulations, we conclude that under these conditions, the change in width, due to multiple scattering, is only $\approx 1 \%$, whereas a $3 \%-4.5 \%$ increase would be required to get good agreement with the theory for diamond, or the NCS data of Ref. 2 for HOPG.

There were only 2014 trajectories where the electrons scattered from $\mathrm{C}$ and $\mathrm{Au}$ atoms. The corresponding spectrum was, considering its poor statistics, not distinguishable from that of electrons scattered from $\mathrm{C}$ atoms only. Thus, in this class, the deflections from an $\mathrm{Au}$ atom was dominated by small-angle deflections .

Multiple scattering has been studied for NCS as well. ${ }^{29}$ Comparing these simulations with the one described here, we can illustrate clearly two important differences in the nature of multiple scattering between neutron and electron scattering.

The first difference relates to the probing depth. For neutron scattering, the signal obtained is from the whole target. If one chooses the target thicker, then the multiple scattering contribution increases. For electrons, the length of trajectories that contribute to the elastic peak is determined by the inelastic mean free path, as after an inelastic event its intensity is shifted out of the elastic peak energy loss range. If the total trajectory length is several times this mean free path $\approx \approx 60 \mathrm{~nm}$ at $40 \mathrm{keV}$ ), then the chance that such a trajectory contributes to the elastic peak becomes negligibly small. Thus for electrons, except for targets thinner than the inelastic mean free path, the target thickness does not influence the rate of multiple scattering.

The second difference relates to the effect of multiple scattering on the spectrum. For electrons, the peak shape of the single and multiple scattered electrons is very similar. For neutrons, these shapes are rather different. This is a direct consequence of the different nature of the probes. The neutronnucleus interaction is very short ranged. Hence, the scattering intensity distribution is rather isotropic. Large-angle scattering events are as likely as small-angle scattering events, and two large-angle scattering event will have different recoil energies. Hence, the multiple scattering contribution in Ref. 29 have a peak shape that deviates strongly from the single scattering one. For electrons, the interaction with the nucleus is, by a screened Coulomb potential, a long range potential. As a consequence, the scattering intensity distribution is severely forwardly peaked. Multiple scattering trajectories are dominated by a single large-angle and one (or more) small-angle scattering events. ${ }^{26,30}$ The contribution to the recoil energy of the latter are very small. Thus, the peak shapes of single and multiple scattered electrons are very similar. As a consequence, we reach a somewhat surprising conclusion: although multiple scattering is more frequent in the electron experiment compared to the neutron experiment, the influence of 
multiple scattering on the obtained peak shape (and hence its influence on the measurement of the kinetic energy of atoms) is less. The influence of multiple scattering for the neutron case can be made very small by choosing the target extremely thin, but this affects the data acquisition rate. For electrons, it is often not practical to limit multiple scattering by the use of thin samples, as it requires samples much thinner than the inelastic mean free path.

\section{B. Corrections due to the presence of different isotopes}

As pointed out already in Ref. 26, another small source of additional broadening that could have some effect is due to different isotopes. About $1 \%$ of carbon is ${ }^{13} \mathrm{C}$, which has, under our scattering conditions, a recoil energy of $5.99 \mathrm{eV}$ rather than $6.49 \mathrm{eV}$ which applies to ${ }^{12} \mathrm{C}$. The kinetic energy of ${ }^{13} \mathrm{C}$ atoms is $\sqrt{12 / 13}$ of that of ${ }^{12} \mathrm{C}$ atoms ${ }^{7}$ and, using Eq. (4), the intrinsic width of the ${ }^{13} \mathrm{C}$ peak is thus 0.92 times the width of ${ }^{12} \mathrm{C}$. In this way, we can include the effect of the isotope in the fit, without introducing any additional free parameters. The change in intrinsic width for ${ }^{12} \mathrm{C}$ was a reduction by $0.5 \%-$ $0.6 \%$ compared to the fit without considering the presence of different isotopes. This change is of the order of the purely statistical error bar obtained by the fitting procedure for $\sigma$. Again, due to the quadratic nature of Eq. (3), this causes a $1 \%$ reduction in the measured kinetic energy.

The last column of Tables I and II show our estimate of the kinetic energy after corrections for both multiple scattering and the presence of different isotopes.

\section{CONCLUSION}

We have shown that electron scattering can be used to obtain information about the kinetic energies of atoms in solids. The values obtained by electron scattering are (after correction for multiple scattering and isotope effects) within $3 \%$ $6 \%$ with the most recent neutron results for HOPG. ${ }^{2}$ There is good agreement between our angular averaged results and the calculations of Nicklow et al., ${ }^{22}$ and somewhat larger $(\approx 6 \%)$ than the calculated values of Young and Koppel ${ }^{15}$ and AlJishi and Dresselhaus. ${ }^{21}$ There is still a sizable difference in the anisotropy as calculated $\left(T_{p} / T_{c}=1.45\right)$ (Ref. 15) and as measured here $\left(T_{p} / T_{c}=1.88\right)$ or as measured by the most recent neutron experiment $\left(T_{p} / T_{c}=1.93\right) .^{2}$

The present value is also within $6 \%$ with the calculated value for diamond. This spread is small compared to that of the earlier NCS measurements and the results of the NRPS method. The deviation is, however, still larger than the statistical uncertainty which is somewhat surprising as the calculations are straightforward, and the experimental techniques rather direct.

Finally, it should be emphasized that the ECS technique requires relatively modest hardware. Increasing its energy resolution (and hence its ability to measure the momentum distribution of, in particular, heavier elements) is a very realistic possibility. This contrasts with the photon and neutron techniques that rely on the sharpness of resonances of suitable isotopes. Electron scattering is somewhat surface sensitive (probing depth about $30 \mathrm{~nm}$ for $40 \mathrm{keV}$ electrons in carbon) compared to the other techniques, but this is very bulk sensitive by surface science standards. This means that some influence of surface effects could affect its result, but also opens up the possibility of studying momentum distributions in thin films.

\section{ACKNOWLEDGMENTS}

We would like to thank Dr. J. E. Butler from the Naval Research Center in Washington DC for the CVD diamond sample used in the ECS measurements and Professor Weigold for critically reading the manuscript. This work is made possible by a grant of the Australian Research Council.

${ }^{1}$ G. I. Watson, J. Phys.: Condens. Matter 8, 5955 (1996).

${ }^{2}$ A. L. Fielding, D. N. Timms, and J. Mayers, Europhys. Lett. 44, 255 (1998).

${ }^{3}$ H. Rauh and N. Watanabe, Phys. Lett. 100A, 244 (1984).

${ }^{4}$ M. P. Paoli and R. S. Holt, J. Phys. C 21, 3633 (1988).

${ }^{5}$ J. Mayers, T. M. Burke, and R. J. Newport, J. Phys.: Condens. Matter 6, 641 (1994).

${ }^{6}$ R. Moreh, O. Beck, D. Jäger, Y. Finkelstein, U. Kneissl, J. Margraf, H. Maser, and H. H. Pitz, Phys. Rev. B 56, 187 (1997).

${ }^{7}$ Y. Finkelstein, O. Beck, R. Moreh, D. Jäger, U. Kneissl, J. Margraf, H. Maser, and H. H. Pitz, Phys. Rev. B 58, 4166 (1998).

${ }^{8}$ R. Moreh and M. Fogel, Phys. Rev. B 50, 16184 (1994).

${ }^{9}$ D. Nemirovsky, R. Moreh, K. H. Andersen, and J. Mayers, J. Phys.: Condens. Matter 12, 4293 (2000).

${ }^{10}$ M. Vos, Phys. Rev. A 65, 12703 (2001).

${ }^{11}$ M. Vos and M. R. Went, Phys. Rev. B 74, 205407 (2006).

${ }^{12}$ M. Vos, M. R. Went, Y. Kayanuma, S. Tanaka, Y. Takata, and J. Mayers, Phys. Rev. B 78, 024301 (2008).

${ }^{13}$ M. Vos and M. Went, J. Phys. B 42, 065204 (2009).

${ }^{14}$ M. Vos, J. Chem. Phys. 132, 074306 (2010).

${ }^{15}$ J. A. Young and J. U. Koppel, J. Chem. Phys. 42, 357 (1965).

${ }^{16} \mathrm{M}$. Vos, C. A. Chatzidimitriou-Dreismann, T. Abdul-Redah, and J. Mayers, Nucl. Instrum. Methods Phys. Res. B 227, 233 (2004).

${ }^{17}$ M. Went and M. Vos, Nucl. Instrum. Methods Phys. Res. B 266, 998 (2008).

${ }^{18}$ M. Went and M. Vos, Appl. Phys. Lett. 90, 072104 (2007).

${ }^{19}$ J. Butler and A. Sumant, Chem. Vap. Deposition 14, 145 (2008).

${ }^{20}$ S. Tanuma, C. J. Powell, and D. R. Penn, Surf. Interface Anal. 21, 165 (1994).

${ }^{21}$ R. Al-Jishi and G. Dresselhaus, Phys. Rev. B 26, 4514 (1982).

${ }^{22}$ R. Nicklow, N. Wakabayashi, and H. G. Smith, Phys. Rev. B 5, 4951 (1972).

${ }^{23}$ W. Weber, Phys. Rev. B 15, 4789 (1977).

${ }^{24}$ G. Dolling and R. A. Cowley, Proc. Phys. Soc. London 88, 463 (1966).

${ }^{25}$ W. A. Kamitakahara, J. Phys. Chem. Solids 57, 671 (1996).

${ }^{26}$ V. Afanas'ev, M. Afanas'ev, A. Lubenchenko, A. Batrakov, D. Efremenko, and M. Vos, J. Electron Spectrosc. Relat. Phenom. 177, 35 (2010).

${ }^{27}$ K. Tökési and D. Varga, Surf. Sci. 604, 623 (2010).

${ }^{28}$ D. Varga, K. Tökési, Z. Berényi, J. Tóth, L. Körvér, G. Gergely, and A. Sulyok, Surf. Interface Anal. 31, 1019 (2001).

${ }^{29}$ J. Mayers, A. L. Fielding, and R. Senesi, Nucl. Instrum. Methods Phys. Res. A 481, 454 (2002).

${ }^{30}$ F. Yubero, N. Pauly, A. Dubus, and S. Tougaard, Phys. Rev. B 77, 245405 (2008). 